\title{
621.928.37:532.5.031
}

ТАНГЕНЦИАЛЬНАЯ СКОРОСТЬ В ГИДРОЦИКЛОНЕ

\section{THE TANGENTIAL VELOCITY IN THE HYDROCYCLONE}

\author{
Капустин Р.П. \\ Kapustin R.P. \\ Брянский государственный инженерно-технологический университет (Брянск. Россия) \\ Bryansk state University of engineering and technology (Bryansk, Russian Federation)
}

\begin{abstract}
Аннотация. В статье на основе уравнения НавьеСтокса приведен вывод формульг тангенциальной скорости потока в гидроииклоне. Вывод совпадает с решением задачи о диффузии вихревой нити. Формула отражает влияние на тангенцииальную скорость геометрических и энергетических параметров гидрочиклона. Приведены гра-фики, показывающиче изменение скорости по высоте аппарата. Приведен график влияния на тангенцииальную скорость вязкости разделяемой среды. В коническом гидроичиклоне с вихревым ичиркуляциионным кольцом тангенцииальная скорость выше кольца увеличивается за счёт добавления составляющей скорости вихревого кольца. На уровне плоскости кольца она значительно уменьшается, ниже плоскости кольца может иметь обратное направление, затем кривые тангенциальной скорости при приближении к нижнему стоку в осевой части значительно возрастают, воспринимая дополнительно воздействие вихревой воронки стока. У цилиндрического гидроциклона с осевой разгрузкой, у которого радиальные скорости у стенки аппарата равны нулю, тангенциальная скорость в верхней части гидрочиклона практически постоянная. Увеличение и всплеск скорости наблюдается только у нижнего разгрузочного отверстия из-за воздействия вихревой воронки стока. В прямоточном иилиндрическом гидрочиклоне около разгрузочных отверстий радиальные скорости имеют разное направление, и тангенциальная скорость около них также может иметь обратное направление. Для устранения этого явления в формуле тангенциальной скорости необходимо использовать абсолютной значение показателя степени экспоненты. При наличии нескольких разгрузочных отверстий одинаковая тангенциальная скорость около этих отверстий будет при равенстве расходов через эти отверстия.
\end{abstract}

Ключевые слова: гидроциклон, тангенцииальная скорость, радиальная скорость, вязкость, вихревое кольичо.

Дата принятия к публикации: Дата публикации:

Сведения об авторе:

Капустин Родион Петрович - кандидат технических наук, доцент, e-mail: k-rodion37@mail.ru

\begin{abstract}
Based on the Navier-Stokes equation, the paper presents the derivation of the tangential flow velocity formula in a hydrocyclone. The derivation coincides with the solution of the vorte $3 x$ filament diffusion problem. The formula reflects the influence on the tangential velocity of the geometric and energy parameters of the hydrocyclone. The graphs showing the change in speed along the height of the device are given. A graph of the effect of velocity of the viscosity of the separated medium is given. In a conical hydrocyclone with a vortex circulation ring, the tangential velocity above the ring is increased by adding the velocity component of the vortex ring. At the level of the plane of the ring is reduced considerably, below the plane in this ring it might have the opposite direction, then peripheral sped curves, when approaching the low in the axial part, are significantly increased, taking the additional effect of vortex flow. In a cylindrical hydrocyclone with axial discharge, in with the radial velocities at the wall of the apparatus equal zero, the tangential velocity at the top of the hydrocyclone is almost constant. The increase and surge in velocity is observed only at the lower discharge opening due to the impact of the vortex funnel of the drain. In a flowing cylindrical hydrocyclone near the discharge holes, the radial velocities have different directions, and the tangential velocity near them may also have a reverse direction. To eliminate this phenomenon, the absolute value of the exponential exponent must be used in the tangential velocity formula. If there are several discharge holes, the same tangential velocity near these holes will be equal if the flow through these holes is equal.
\end{abstract}

Keywords: hydrocyclone, tangential velocity, radial velocity, viscosity, vortex ring.

Date acceptance for publication:

26.02 .2020 Date of publication:

25.06.2020

Author' information:

Rodion P. Kapustin - Candidate of Technical Sciences, Associate Professor, e-mail: k-rodion37@mail.ru 


\section{1. Введение}

Определяющее значение на процесс разделения продукта в гидроциклоне оказывает тангенциальная скорость движения разделяемого продукта.

Формулы тангенциальной скорости, приводимые в научной и прикладной литератуpe, в большинстве случаев основаны на результатах экспериментальных данных, имеют приближенный характер, не отражают влияния на неё вязкости продукта и геометрических параметров гидроциклона.

Kelsall D.F. установил [1], что для любой точки гидроциклона соотношение между тангенциальной скоростью и радиусом вращения справедливо равенство:

$$
V r^{n}=\text { const, }
$$

где $V$-тангенциальная скорость; $r$-радиус вращения; $n$-показатель степени.

Фонтейн и Диксма [2] по результатам экспериментов предложили зависимость для тангенциальной скорости

$$
V=\frac{C_{1}}{r}+C_{2} r
$$

где $\mathrm{C}_{1}$ и $\mathrm{C}_{2}$ - коэффициенты, зависящие от конструкции гидроциклона и числа Рейнольдса.

Жангарин А.И. для низконапорного гидроциклона тангенциальную скорость предлагает определять по формуле [3]

$$
V=V_{\theta x} \sqrt[3]{\left(\frac{r_{u}}{r_{x}}\right)^{2}},
$$

где $r_{u}$-радиус цилиндрической части гидроциклона; $r_{x}$-радиус вращения; $V_{\theta x}$-средняя скорость потока на входе.

Лилдж [4] подтвердил зависимость тангенциальной скорости от радиуса вращения и предложил для её определения следующее выражение:

$$
V=m r+b,
$$

где $m$ и $b$ - коэффициенты, зависящие от горизонтального уровня гидроциклона, на котором определяются скорости.

Литовко В.И. [5] подтвердил переменность показателя $n$ в формуле Келсалла от радиуса вращения и горизонтального уровня гидроциклона. Предложенная им формула имеет вид

$$
V=\frac{A e^{r / h}+B e^{-r / h}}{r},
$$

где $V$-тангенциальная скорость на радиусе вращения; $A, B$-коэффициенты, зависящие от параметров гидроциклона (радиуса вращения $r$ и расстояния $h$ от оси питающего патрубка).

Деметр [6] читает тангенциальную скорость постоянной, равной входной, которую предлагает определять по формуле:

$$
V_{в x}=\sqrt{\frac{2 g H}{1+2 \lg \frac{R_{K}}{R_{B}}}},
$$

где $V_{\text {вx }}$-скорость жидкости на входе в гидроциклон; $R_{K}$ и $R_{B}-$ внешний и внутренний радиусы потока жидкости в гидроциклоне; $2 g H$ - давление потока на входе.

Как следует из анализа, мнения исследователей по характеру изменения тангенциальной скорости в гидроциклоне различны, являются результатом экспериментов и применимы к ограниченному типоразмеру гидроциклонов. Эта тенденция продолжается и в настоящее время [7].

Большинство исследователей использу-ют формулу Келсалла [1], подбирая под фо-рмулу показатель степени $n$. При таком изъяснении результатов экспериментов не учитываются геометрические и энергетичесие параметры гидроциклона. Из этого вытекает необходимость теоретическим методом исследования влияния разных факторов на тангенциальную скорость потока в гидроциклоне.

\section{2. Теоретические исследования}

Будем основываться на условии, что движение потока в гидроциклоне симметричное и установившееся, т.е.

$$
\frac{d U}{d t}=\frac{d W}{d t}=\frac{d V}{d t}=0 .
$$

где $U, W, V$ - радиальная, осевая и тангенциальная скорости.

Для исследования примем второе уравнение системы Навье-Стокса. В нём радиальная и осевая скорости взаимосвязаны: 


$$
\begin{gathered}
U \frac{d V}{d r}+U \frac{V}{r}+W \frac{d V}{d z}= \\
=v\left(\frac{d^{2} V}{d r^{2}}+\frac{1}{r} \frac{d V}{d r}-\frac{V}{r}+\frac{d^{2 V}}{d z^{2}}\right) ; \\
U \frac{d V}{d r}+U \frac{V}{r}-v\left(\frac{d^{2} V}{d r^{2}}+\frac{1}{r} \frac{d V}{d r}-\frac{V}{r}\right)= \\
=v \frac{d^{2} V}{d z^{2}}-W \frac{d V}{d z} .
\end{gathered}
$$

Приравняем левую часть уравнения (3) нулю

$$
U \frac{d(V r)}{r d r}-v \frac{d}{d r}\left[\frac{d(V r)}{r d r}\right]=0 .
$$

Введём обозначения

$$
r=x ; \quad d r=d x ; \quad \frac{d(V r)}{r d r}=y .
$$

Тогда уравнение примет вид

$$
U y-v \frac{d y}{d x}=0, \quad \text { или } \frac{1}{v} U d x-\frac{d y}{y}=0 .
$$

Далее будем иметь

$$
\begin{gathered}
\frac{U}{v} d r=\ln \left(\frac{y}{C_{1}}\right), \\
y=C_{1} e^{-\frac{U}{v} d r} \quad \frac{d(V r)}{r d r}=C_{1} e^{-\frac{U}{v} d r} ; \\
V r=C_{1} \int_{0}^{r} e^{-\frac{U}{v} d r} r d r+C_{2} .
\end{gathered}
$$

При $r=0$ имеем $V=0$,

$$
C_{1} \int_{0}^{r} e^{-\frac{U}{V} d r} r d r=0
$$

следовательно, $C_{2}=0$, тогда

$$
V r=C_{1} \int_{0}^{r} e^{-\frac{U}{v} d r} r d r
$$

Для решения полученного уравнения следует подставить выражение радиальной скорости $U$. Поскольку выражение радиальной скорости сложное, решение уравнения (6) становится практически невозможным, поэтому заменим его простым:

$$
U=-8 r,
$$

где $в$ - коэффициент коррекции.

Как известно, зона наиболее эффективного разделения находится на уровне входной кромки верхнего выходного патрубка, то коэффициент коррекции можно определить по формуле:

$$
B=\frac{U_{\max 8 n}}{r_{n}},
$$

где $U_{\max \text { вn }}$-максимальная радиальная скорость потока на уровне верхнего сливного патрубка; $r_{n}$-радиус циклона на уровне входной кромки верхнего сливного патрубка.

Тогда для гидроциклона имеем

$$
V r=C_{1} \int_{0}^{r} e^{-\frac{\theta}{2 v} r^{2}} r d r=\frac{v}{B} C_{1}\left(1-e^{-\frac{\theta}{2 v} r^{2}}\right) .
$$

Решение совпадает с решением задачи о диффузии вихревой нити [8].

Постоянную $C_{1}$ определим из условия, что на входе в гидроциклон $\left(r=r_{c}\right)$ тангенциальная скорость равна начальной $V=V_{0}$, или

$$
C_{1}=\frac{\beta V_{0} r_{c}}{v\left(1-e^{-\frac{\beta}{2 v} r_{c}^{2}}\right)} .
$$

В результате получим

$$
V=\frac{V_{0} r_{c}}{r} \frac{\left(1-e^{-\frac{\beta}{2 v} r^{2}}\right)}{\left(1-e^{-\frac{\beta}{2 v} r_{c}^{2}}\right)} .
$$

B работе [9] Rott N. предложил подобную формулу для вязкого вихря без знаменателя от экспоненты. Поскольку эта величина постоянная и достаточно мала, то формулу (10) можно упростить и представить в виде:

$$
V=\frac{V_{0} r_{c}}{r}\left(1-e^{-\frac{6}{2 v} r^{2}}\right) .
$$

Оценку формулы проведём для конического гидроциклона [1]: $D=7,5 \mathrm{~cm} ; z_{c}=28 \mathrm{cm;}$ $r_{c}=75 \mathrm{~cm} ; z_{n}=17 \mathrm{~cm} ; r_{n}=1,25 \mathrm{~cm} ; \beta=10^{\circ}$; $q_{c m}=399 \mathrm{~cm}^{3} / \mathrm{c} ; r_{0}=0,5 \mathrm{~cm} ; r_{c m}=0,5 \mathrm{~cm}, z_{c m}=$ $2,8 \mathrm{~cm} ; V_{0}=500 \mathrm{~cm} / \mathrm{c} ; \quad в=203 \mathrm{~cm} / \mathrm{c} \quad$ при $v=1 \ldots 65 \mathrm{cC}$.

Характер кривых, показанных на рис. 1, соответствует реальным, приведенным в [10] и в других источниках, а потому предлагаемая формула при введении соответствующего корректирующего коэффициента может быть использована для практических расчётов. 


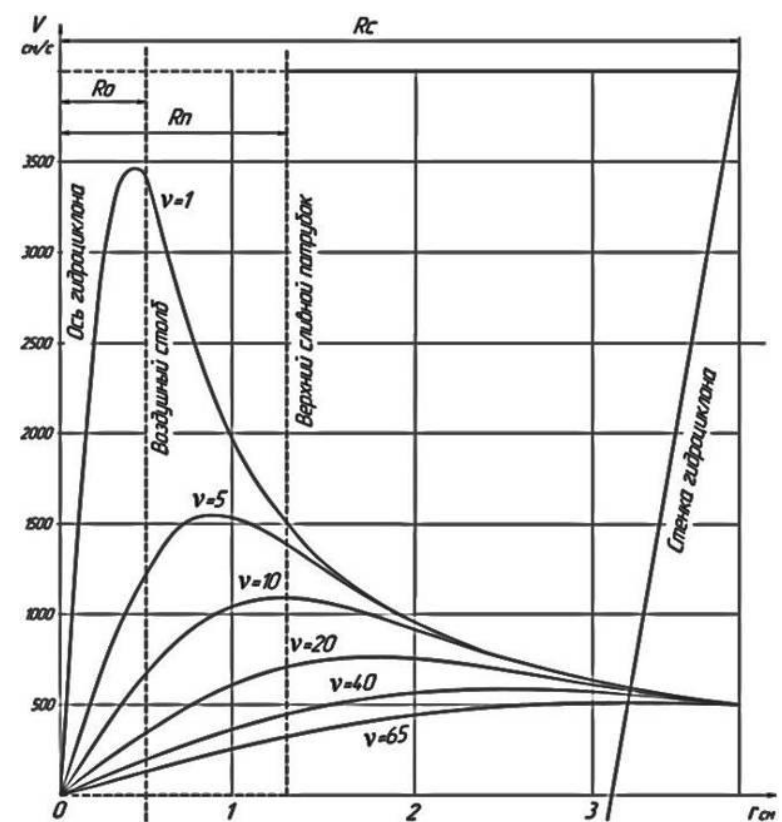

Рис.1. Зависимость тангенциальной скорости потока в коническом гидроциклоне от вязкости разделяемого продукта $v$

В гидроциклоне по мере осветления разделяемого продукта вязкость первоначальная на входе в аппарат с уменьшением радиуса вращения уменьшается, следовательно, скорость вращения будет увеличиваться, хотя это будет незаметно.

В формуле (12) выражение $6 r^{2}$ практически описывает характер изменения радиальной скорости потока в гидроциклоне, что позволяет это выражение заменить на действительное выражение радиальной скорости, т.е.

$$
V=\frac{V_{0} r_{c}}{r}\left(1-e^{-\frac{u}{v}}\right),
$$

где $u$-радиальная скорость потока в гидроциклоне.

Это даёт возможность оценить влияние энергетических и конструктивных параметров гидроциклона на тангенциальную скорость потока.

На рис. 2 показан характер изменения тангенциальной скорости в коническом гидроциклоне с параметрами, приведенными выше при отсутствии и наличии вихревого циркуляционного кольца [11].

В гидроциклоне вихревое циркуляционное кольцо существенно изменяет характер тангенциальной скорости. Выше кольца тангенциальная скорость увеличивается за счёт добавления составляющей скорости вихревого кольца. На уровне плоскости кольца скорость значительно уменьшается и может иметь обратное направление, затем кривые тангенциальной скорости при приближении к нижнему стоку принимают естественный вид и в осевой части значительно возрастают, воспринимая дополнительное воздействие вихревой воронки стока.
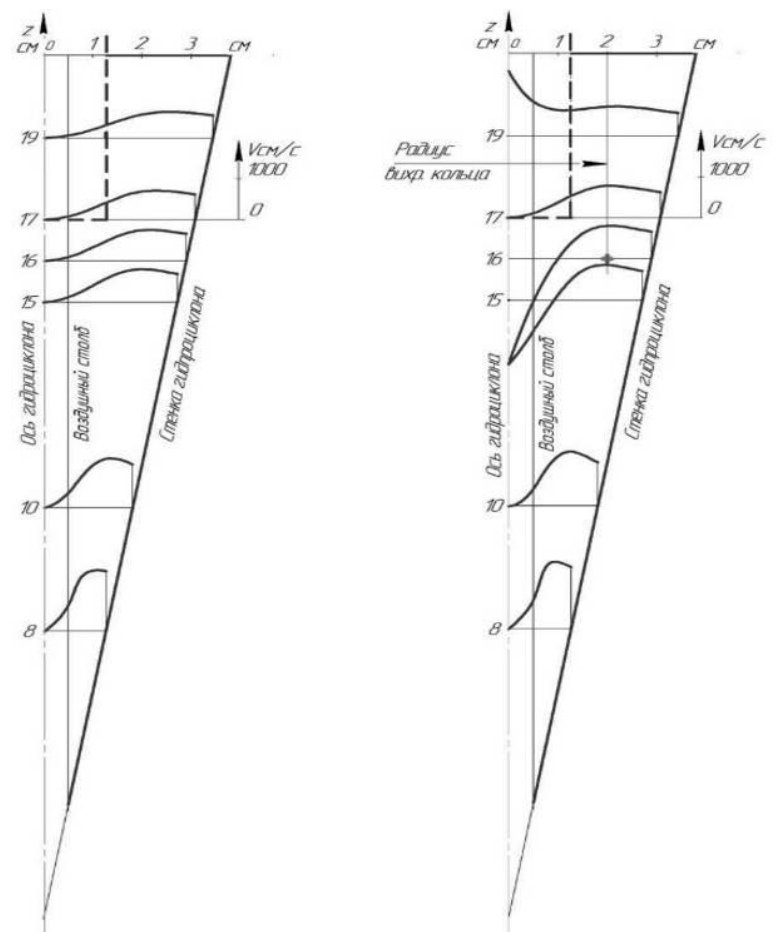

Рис. 2. Распределение тангенциальных скоростей в коническом гидроциклоне при отсутствии и наличии вихревого кольца

Цилиндрический гидроциклон с осевой разгрузкой характер тем, что у него радиальные скорости у стенки равны нулю [12]. С уменьшением радиуса вращения они увеличиваются, достигая максимального значения, примерно, около нулевого значения осевой скорости, плавно уменьшаясь до нуля на оси гидроциклона или на границе воздушного столба. Это сказывается на распределении тангенциальной скорости внутри аппарата (рис. 3, 4).

Всплеск скорости наблюдается только у нижнего разгрузочного отверстия, где сказывается воздействие вихревой воронки стока.

В прямоточном цилиндрическом гидроциклоне, описанном в работах $[10,13]$, имеются три разгрузочных отверстия. Расчетные 


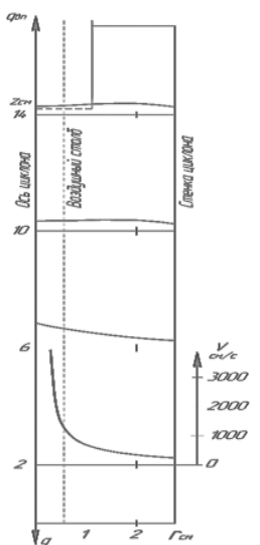

Рис. 3. Характер изменения тангенциальной скорости потока в цилиндрическом гидроциклоне ( $D=5,5 \mathrm{~cm} ; H=15,5 \mathrm{~cm}$; $r_{n}=1,2 \mathrm{~cm} ; r_{0}=0,5 \mathrm{~cm} ; z_{n}=14,2 \mathrm{~cm} ;$ $\left.q_{\text {вn }}=3667 \mathrm{~cm}^{3} / \mathrm{c} ; q=1667 \mathrm{~cm}^{3} / \mathrm{c} ; V_{0}=500 \mathrm{~cm} / \mathrm{c}\right)$

радиальные скорости в нём около разгрузочных отверстий имеют разное направление, что приводит к изменению направления тангенциальной скорости, точнее, появлению обратного вихря, поэтому для наглядности на графике в формуле (12) должно использоваться абсолютное значение скорости, т.е.

$$
V=\frac{V_{0} r_{c}}{r}\left(1-e^{-\frac{a b s(U)}{v}}\right) .
$$

Из графиков видно, что тангенциальная скорость около разгрузочных отверстий будет практически одинаковой, если расходы между ними будут равными. Таковым является четвертый вариант. Представленные графики хорошо согласуются с экспериментальными [10].

\section{3. Выводы}

Для рассмотренных вариантов гидроциклонов предложенные формулы для определения тангенциальной скорости повторяют реальный характер её изменения.

Появление в гидроциклоне вихревого циркуляционного кольца резко влияет на изменение тангенциальной скорости и способствует возникновению обратного вихря. Эти факторы могут положительно или отрицательно влиять на эффективность работы гидроциклона. Обнаруженные факторы требуют дополнительного экспериментального исследования.

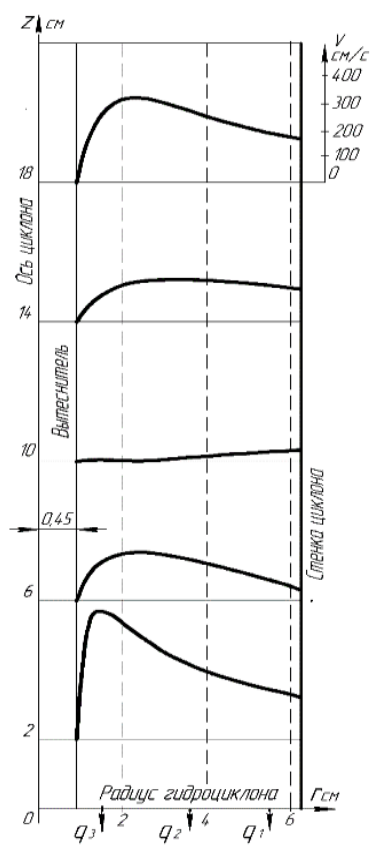

B)

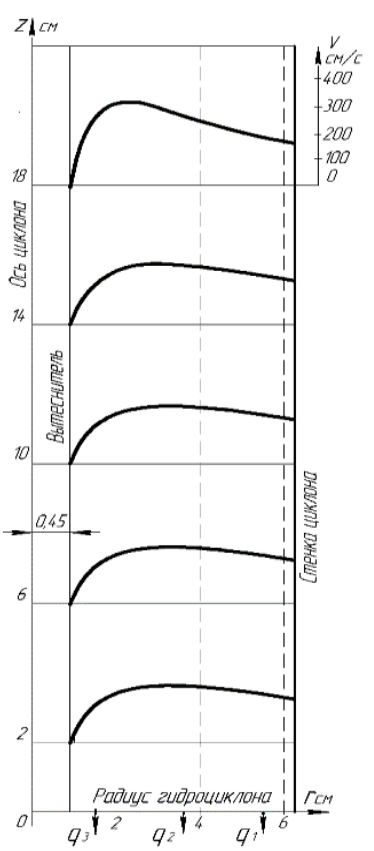

г) б)

Рис. 4. Распределение тангенциальных скоростей в прямоточном цилиндрическом гидроциклоне: $1-q_{1}=128 \mathrm{~cm}^{3} / \mathrm{c} ; q_{2}=133 \mathrm{~cm}^{3} / \mathrm{c} ; q_{3}=850 \mathrm{~cm}^{3} / \mathrm{c} ; 2-q_{1}=128 \mathrm{~cm}^{3} / \mathrm{c} ; q_{2}=850$ $\mathrm{cm}^{3} / \mathrm{c} ; q_{3}=133 \mathrm{~cm}^{3} / \mathrm{c} ; 3-q_{1}=850 \mathrm{~cm}^{3} / \mathrm{c} ; q_{2}=133 \mathrm{~cm}^{3} / \mathrm{c} ; q_{3}=128 \mathrm{~cm}^{3} / \mathrm{c} ; 4-q_{1}=q_{2}=q_{3}$ 


\section{Список литературы}

1. Kelsall D.F. Study of the Motion Solid Particles in a Mineral Dressing // The institution of Mining and Metallurgy. 1953. P. 209-227.

2. Применение гидроциклонов при обогащении углей // Сб. переводов статей / Под ред. В.Г. Черненко. М.: Госгортехиздат, 1960. 162 c.

3. Жангарин А.И. К вопросу гидравлического расчёта гидроциклона // Вестник АНКазССР, 1960. №10 (211). С.55-64.

4. Мустафаев А.М., Гутман Б.М. Теория и расчёт гидроциклона. Баку: Маариф, 1969. $172 \mathrm{c}$.

5. Классен В.И., Литовко В.И. Некоторые вопросы разделения минеральных зёрен в водной среде // Научное сообщение АН ИГД. 1960. Вып. IV. C. 38-45.

6. Акопов М.Г. Основы обогащения углей в гидроциклонах. М.: Недра, 1967. 179 с.

7. Валеев С.И., Булкин В.А. Гидродинамика цилиндрического гидроциклона с удлинённым верхним сливным патрубком // Вестник технологического университета. 2015. Е.18. C. 231-232.

8. Лойцянский Л.Г. Механника жидкости и газа. М.: Наука, 1978. 736 с.

9. Rott N. On the Viscous Core of a Line Vortex // Math. und Phys. 1958. 9b. №5-6. S. 53-55.

10. Терновский И.Г. Гидроциклонирование. М.: Наука, 1994. 352 с.

11. Капустин Р.П. Конический и цилиндроконический гидроциклоны. Анализ кинематики потока // Актуальные проблемы гуманитарных и естественных наук. 2018. №6. C. 20-27.

12. Капустин Р.П. Радиальные и осевые скорости жидкости в цилиндрическом гидроциклоне // Обогащение руд. 2013. №1. С. 23-26.

13. Капустин Р.П. Анализ кинематики потока в прямоточном цилиндрическом гидроциклоне // Всероссийский научный вестник. 2016. №6. С. 61-65.

\section{References}

1. Kelsall D.F. Study of the Motion Solid Particles in a Mineral Dressing. The Institution of Mining and Metallurgy, 1953, pp. 209-227.

2. Primenenie gidrotsiklonov pri obogashchenii ugley. Moscow, Gosgortekhizdat, 1960. 162 p. (In Russian).

3. Zhangarin A.I. K voprosu gidravliceskogo rascheta gidrotsiklona. Vestnik $A N$ KazSSR, 1960, №10 (211), pp. 55-64. (In Russian).

4. Mustafaev A.M., Gutman B.M. Teoriya $i$ raschet gidrotsiklona. Baku, Maarif, 1969. 172 p. (In Russian).

5. Klassen V.I., Litovko V.I. Nekotorye voprosy razdeleniya mineralnykh zeren $\mathrm{v}$ vodnoy srede. Nauchnoe soobshchenie AN IGD, 1960, Vol. IV, pp. 38-45. (In Russian).

6. Akopov M.G. Osnovy obogashcheniya ugley $v$ gidrotsiklonakh. Moscow, Nedra, 1967. 179 p. (In Russian).

7. Valeev C.I., Bulkin V.A. Gidrodinamika tsilindricheskogo gidrotsiklona $\mathrm{s}$ udlinennym verkhnim slivnym patrubkom. Vestnik tekhnologicheskogo universiteta, 2015, Vol.18, pp. 231-232. (In Russian)

8. Loytsynskiy L.G. Mekhanika zhidkosti i gaza. Moscow, Nauka, 1994. 736 p. (In Russian).

9. Rott N. On the Viscous Core of a Line Vortex. Math. und Phys., 1958, No.56, pp. 53-55.

10. Ternovskiy I.G. Gidrotsiklonirovanie. Moscow, Nauka,1994. 352 p. (In Russian).

11. Kapustin R.P. Konicheskiy i tsilidrokonicheskiy gidrotsiklony. Analiz kinematiki potoka. Aktualnye problemy gumanitarnykh i estestvennnykh nauk, 2018, No.6, pp. 20-27. (In Russian)

12. Kapustin R.P. Radialnye i osevye skorosti $\mathrm{v}$ tslindricheskom gidrotsiklone. Obogaschenie rud, 2013, No.1, pp. 23-26. (In Russian).

13. Kapustin R.P. Analiz kinematiki potoka $\mathrm{V}$ pryamotochnom tsilindricheskom gidrotsiklone. Vserossiyskiy nauchnyiy vestnik, 2016, No.6, pp. 61-65. (In Russian). 